\title{
RELAÇÃO ENTRE ESTILO DE VIDA E DESEMPENHO ACADÊMICO
}

\author{
Relationship between lifestyle and academic performance \\ Relación entre el estilo de vida y el rendimiento académico
}

\author{
Quelen Schutz Carvalho Bernardes Malafaia (iD \\ Universidade Regional de Blumenau - FURB - Blumenau (SC) - Brasil \\ Cristiani Devalieri iD \\ Universidade Regional de Blumenau - FURB - Blumenau (SC) - Brasil \\ Maiara Vargas Schmitt iD \\ Universidade Regional de Blumenau - FURB - Blumenau (SC) - Brasil \\ Clóvis Arlindo de Sousa \\ Universidade Regional de Blumenau - FURB - Blumenau (SC) - Brasil
}

\section{RESUMO}

Objetivo: Investigar a associação do estilo de vida com o desempenho acadêmico de estudantes de Educação Física. Métodos: Estudo transversal envolvendo 186 estudantes de Educação Física de uma universidade de Santa Catarina, Brasil. Realizou-se a coleta de dados, entre agosto e novembro de 2018, com o questionário "Estilo de Vida Fantástico", composto por 25 questões em nove domínios (família e amigos/ atividade física/ nutrição/ cigarro e drogas/ álcool/ sono, cinto de segurança, estresse e sexo seguro/ tipo de comportamento/ introspecção/ trabalho). Para comparação entre estilo de vida e o desempenho acadêmico, representado pela média semestral, utilizou-se a análise de variância e o coeficiente de correlação de Pearson. Resultados: Verificou-se a média de idade de 22,3 anos $( \pm 4,87)$. O estilo de vida dos estudantes se apresentou, de forma geral, bom $(34,1 \%)$ e muito bom $(48,6 \%)$, e associado de forma positiva fraca $(r=0,232)$, mas significativa $(p=0,003)$, com o desempenho acadêmico. As médias semestrais de desempenho acadêmico diferenciaram-se em relação à classificação do estilo de vida $(p=0,012)$. Os domínios família e amigos, cigarros e drogas, e álcool obtiveram pontuação satisfatória para um estilo de vida saudável. Conclusão: O estilo de vida dos acadêmicos de Educação Física avaliados é bom/muito bom nos domínios estudados e associa-se ao desempenho acadêmico.

Descritores: Estilo de Vida; Estudantes; Desempenho Acadêmico; Educação Física e Treinamento; Promoção da Saúde.

\section{ABSTRACT}

Objective: To investigate the association of lifestyle with the academic performance of Physical Education students. Methods: it is a cross-sectional study consisting of 186 Physical Education students from a university in Santa Catarina, Brazil. Data were collected between August and November 2018 with the "Fantastic Lifestyle" questionnaire, consisting of 25 questions in nine domains (family and friends / physical activity/nutrition/ smoking and drugs/alcohol/ sleep, safety belt, stress, and safe sex/ behavior type/introspection/ work). We used the analysis of variance and Pearson's correlation coefficient to compare lifestyle and academic performance, represented by the semester average. Results: The mean age was 22.3 years ( \pm 4.87$)$. Students' lifestyles were generally good (34.1\%) and very good (48.6\%), and associated positively weak $(r=0.232)$ but significant $(p=$ 0.003), with academic performance. Semiannual averages of academic performance differed to lifestyle classification ( $p=0.012)$. The domains family and friends, cigarettes and drugs, and alcohol scored satisfactorily for a healthy lifestyle. Conclusion: The lifestyle of the evaluated Physical Education students is good / very good in the studied domains and is associated with academic performance.

Descriptors: Life Style; Students; Academic Performance; Physical Education and Training; Health Promotion.

\section{RESUMEN}

Objetivo: Investigar la asociación entre el estilo de vida y el rendimiento académico de estudiantes de Educación Física. Métodos: Estudio transversal con 186 estudiantes de Educación Física de una universidad de Santa Catarina, Brasil. La recogida de datos 
se dio entre agosto y noviembre de 2018 con el cuestionario "Estilo de Vida Fantástico" con 25 preguntas de nueve dominios (familia y amigos/ actividad física/ nutrición/ cigarro y drogas/ alcohol/ sueño, cinturón de seguridad, estrés y sexo seguro/ tipo de conducta/ introspección/ trabajo). Se utilizó el análisis de la varianza y el coeficiente de correlación de Pearson para la comparación entre el estilo de vida y el rendimiento académico representado por la media de cada semestre. Resultados: Se verifico la media de edad de 22,3 años $( \pm 4,87)$. En general, el estilo de vida de los estudiantes ha sido bueno (34,1\%) y muy bueno $(48,6 \%)$ con asociación débil positiva $(r=0,232)$ aunque significativa $(p=0,003)$ con el rendimiento académico. Las medias semestrales de rendimiento académico se han diferenciado de la clasificación del estilo de vida $(p=0,012)$. Los dominios familia y amigos, cigarros y drogas, y alcohol tuvieron puntuación satisfactoria para un estilo de vida saludable. Conclusión: El estilo de vida de los académicos de Educación Física evaluados es bueno/muy bueno para los dominios estudiados y se asocia con el rendimiento académico.

Descriptores: Estilo de Vida; Estudiantes; Rendimiento Académico; Educación y Entrenamiento Físico; Promoción de la Salud.

\section{INTRODUÇÃO}

A Organização Mundial da Saúde define o estilo de vida (EV) como o resultado de padrões de comportamento que o indivíduo desenvolve, determinados por diversos fatores, sendo pessoais, econômicos e ambientais, que acarretam considerável efeito na saúde das pessoas ${ }^{(1,2)}$. Esses fatores são modificáveis e podem contribuir de forma negativa ou positiva para a saúde. Alguns fatores positivos para saúde são a alimentação saudável, nível suficiente de atividade física, manejo do estresse, tempo de sono, atividades de lazer e a satisfação com o trabalho e o estudo ${ }^{(3)}$. Apesar de todas as informações e evidências sobre o tema, há um aumento dos índices de hábitos considerados de risco entre estudantes universitários ${ }^{(2)}$.

Parte do EV é estabelecido na juventude, podendo influenciar na vida adulta. A juventude se relaciona com a vida acadêmica e representa uma fase de mudanças de hábitos na vida dos indivíduos. É também nessa fase que se pode observar maus hábitos alimentares, sedentarismo, bem como hábitos noturnos (com frequentes festas e barzinhos) e maior frequência de consumo de bebidas alcoólicas, ocasionando prejuízos à saúde e ao desempenho acadêmico( ${ }^{(3)}$.

O desempenho acadêmico é compreendido como o grau de competências de um indivíduo em um determinado nível educacional. Dizer que um estudante tem um bom desempenho acadêmico significa atestar que ele progrediu em termos de conhecimentos, habilidades e atitudes pessoais e sociais para um determinado nível educacional, aspectos necessários para progressão satisfatória na vida acadêmica, social e profissional ${ }^{(4)}$. Diferentes medidas de desempenho acadêmico vêm sendo apresentadas. A média do período (anual, semestral, bimestral) também pode ser utilizada como medida de desempenho. Ela resulta da utilização das notas das disciplinas divididas pela quantidade de matérias cursadas, que resulta em um indicador ao final do período e é um reflexo da performance do acadêmico em todas as disciplinas cursadas naquele período ${ }^{(5,6)}$.

Espera-se que os acadêmicos de Educação Física tenham um EV saudável devido à relação do curso com os esportes e disciplinas que tratam de saúde, mas os estudos encontrados são contraditórios, não só no curso de Educação Física, mas nos cursos da área da saúde em geral ${ }^{(7,8)}$.

Um estudo realizado com estudantes de Educação Física de Sergipe mostrou um nível de EV inadequado, quando mais da metade dos entrevistados não atendeu às recomendações para atividade física. Destacam-se, como componentes do EV mais inadequados, a falta de sono, o não uso de cinto de segurança, o estresse e o sexo inseguro (7). Outra pesquisa, com acadêmicos de Educação Física no Paraná, apresentou como resultado geral um EV adequado, mas com domínios mais inadequados, como o tipo de comportamento, a nutrição e a prática de atividade física ${ }^{(2)}$. Estudo realizado com acadêmicos de enfermagem no Amazonas encontrou um nível de EV global satisfatório. O nível de atividade física apresentou um fator importante para o desenvolvimento de um EV saudável e um bom desempenho acadêmico(6).

Os estudantes universitários podem apresentar uma série de comportamentos de risco à saúde. Portanto, o ambiente universitário parece ser um local apropriado para promoção da saúde, uma vez que a melhora do estilo de vida desses indivíduos por meio de ações de promoção da saúde e prevenção de doenças influenciam as condições de vida, os fatores sociais e a saúde dos jovens, atualmente e no futuro. Além dos benefícios na saúde e na qualidade de vida, o EV saudável pode influenciar a produtividade e a vida universitária, o que pode impactar na vida futura dos trabalhadores, para serem mais saudáveis, mais produtivos, com maior capacidade de decisão, menor frequência de doenças relacionadas ao estilo de vida não saudável e menor uso do sistema de saúde ${ }^{(9-12)}$. 
Nesse sentido, o presente estudo buscou investigar a associação do estilo de vida com o desempenho acadêmico de estudantes de Educação Física.

\section{MÉTODOS}

Trata-se de um estudo transversal com amostra composta por estudantes do curso de Educação Física da Universidade Regional de Blumenau (FURB). A FURB é uma instituição pública municipal de ensino superior localizada na cidade de Blumenau, em Santa Catarina. Blumenau está localizada na região do Vale do Itajaí e possui uma população estimada, para 2019 , em 357.199 pessoas. É a terceira cidade mais populosa do estado, constituindo um de seus principais polos industrial, tecnológico e universitário. Seu índice de desenvolvimento humano municipal é de 0,806 (considerado muito alto), ocupando a $25^{a}$ posição entre os municípios brasileiros ${ }^{(13)}$.

O tamanho da amostra mínimo representativo envolveu 169 acadêmicos do curso de Educação Física na FURB. Considerou-se, para a presente pesquisa, a população de 298 acadêmicos, sendo $48,6 \%$ ( $n=87$ ) do sexo feminino e $51,4 \%$ ( $n=92)$ do sexo masculino, e também $50 \%$ do curso de bacharelado e de licenciatura, frequência hipotética do fator do resultado na população de 50\% (+/-5), precisão de $5 \%$ e intervalo de confiança de $95 \%$, finalizando a amostra com 186 estudantes (62\% da população).

Compuseram a amostra os estudantes devidamente matriculados nos cursos de bacharelado e licenciatura em Educação Física da FURB, no segundo semestre de 2018, que aceitaram participar desta pesquisa mediante assinatura do Termo de Consentimento Livre e Esclarecido (TCLE). Excluíram-se sete participantes do estudo pelo preenchimento incorreto do questionário (incorretos ou incompletos).

A coleta de dados ocorreu no período de agosto a novembro de 2018, com a aplicação do questionário Estilo de Vida Fantástico ${ }^{(9)}$, além de perguntas adicionais, referentes à idade, ao sexo, ao semestre atual, ao período (matutino ou noturno) e ao curso (licenciatura ou bacharelado). Durante as aulas, com consentimento do professor responsável, aplicou-se o questionário aos estudantes e realizou-se a coleta da média semestral dos acadêmicos na Divisão de Registro Acadêmico da FURB, que serviu como índice de desempenho acadêmico.

O questionário, traduzido e validado ${ }^{(9)}$ no ano de 2008 , é autoadministrado e tem por objetivo mensurar os principais elementos que caracterizam o EV adequado para a saúde. É uma ferramenta auxiliar utilizada por profissionais da saúde com o propósito de avaliar o EV de estudantes, trabalhadores, pacientes de clínica familiar, pacientes hipertensos, pacientes com diabetes tipo 2 e pessoas da atenção primária ${ }^{(9)}$. É composto por 25 questões distribuídas em nove domínios, sendo eles: 1) família e amigos; 2) atividade física; 3) nutrição; 4) cigarro e drogas; 5) álcool; 6) sono, cinto de segurança, estresse e sexo seguro; 7) tipo de comportamento; 8) introspecção e 9) trabalho. Cada questão possui cinco alternativas, distribuídas em escala de Likert, a qual determina a pontuação que se relaciona com um EV saudável. O questionário tem um escore total de zero a 100. Podendo o indivíduo ser categorizado com um EV excelente (pontuação de 85 a 100), muito bom (pontuação de 70 a 84), bom (pontuação de 55 a 69), regular (pontuação de 35 a 54) ou que necessita melhorar (pontuação de 0 a 34) (9). $^{(2)}$

Para as variáveis quantitativas, utilizaram-se médias e desvios-padrão. Para as variáveis qualitativas, frequências e porcentagens. Para comparação entre a classificação de EV com o desempenho acadêmico representado pela média semestral, utilizou-se a análise de variância (ANOVA). Para análise de associação bivariada entre o EV e o desempenho acadêmico, utilizou-se o coeficiente de correlação de Pearson, considerando-se estatisticamente significativos valores de $p<0,05$.

Este estudo obteve a aprovação do Comitê de Ética em Pesquisa com Seres Humanos da FURB, com o Parecer n. ${ }^{\circ} 2.788 .258 / 2018$.

\section{RESULTADOS}

Dos 186 estudantes avaliados do curso de Educação Física, excluídos os sete que tinham preenchido o questionário de forma inadequada, 179 compuseram a amostra final, dos quais $48,6 \%(n=87)$ eram do sexo feminino e $51,4 \%(n=92)$ do sexo masculino, com idade média de $22,3( \pm 4,87)$ anos. Acadêmicos do curso de bacharelado representam $54,2 \%(n=97)$ da amostra e $45,8 \%(n=82)$ são do curso de licenciatura. Referente ao período de estudo, $44,1 \%(n=79)$ são estudantes do período matutino e 55,9\% $(n=100)$ são do período noturno.

Observa-se que $41,3 \%(n=74)$ dos acadêmicos se encontram entre a primeira e a quarta fase do curso, e $58,7 \%$ $(n=105)$ da quinta a oitava fase. Isto pode ser explicado pelo fato de que não houve abertura de turmas no curso nos semestres anteriores à pesquisa. Esses resultados podem ser observados na Tabela I. 
O sexo feminino obteve a média do escore de EV de 74,1 pontos e o sexo masculino apresentou 71,6 pontos, em uma escala entre zero a 100. A média geral, entre todos os participantes, envolveu 72,7 pontos, o que classifica o EV como muito bom em $48,6 \%$ dos acadêmicos, como dispõe a Tabela II. Apenas sete acadêmicos $(3,9 \%)$ apresentaram a pontuação de EV classificado regular. Encontrou-se o valor mínimo de 38 pontos ( $n=1 ; 1,8 \%)$ para uma acadêmica e 43 pontos $(n=1 ; 1,8 \%)$ para um estudante do sexo masculino. Nenhum acadêmico obteve pontuação em "necessita melhorar" (0 a 34 pontos).

Tabela I - Características dos acadêmicos de Educação Física. Blumenau, Santa Catarina, Brasil, 2018.

\begin{tabular}{lcc}
\hline Características & $\mathbf{n}$ & $\%$ \\
\hline Sexo & 87 & 48,6 \\
Feminino & 92 & 51,4 \\
Masculino & 179 & 100,0 \\
Total & & \\
Graduação & 82 & 45,8 \\
Licenciatura & 97 & 54,2 \\
Bacharelado & 179 & 100,0 \\
Total & & \\
Período & 79 & 44,1 \\
Matutino & 100 & 55,9 \\
Noturno & 179 & 100,0 \\
Total & & \\
Fase & 74 & 41,3 \\
1 a 4 & 105 & 58,7 \\
5 a 8 & 179 & 100,0 \\
Total & & \\
\hline
\end{tabular}

n: número; \%: porcentagem

Tabela II - Classificação do estilo de vida dos acadêmicos de Educação Física. Blumenau, Santa Catarina, Brasil, 2018.

\begin{tabular}{lcc}
\hline Classificação & $\mathbf{n}$ & $\%$ \\
\hline Excelente & 24 & 13,4 \\
Muito bom & 87 & 48,6 \\
Bom & 61 & 34,1 \\
Regular & 7 & 3,9 \\
Total & 179 & 100,0 \\
\hline
\end{tabular}

$\mathrm{n}$ : número; \%: porcentagem

A Tabela III apresenta as médias (escores entre 0 e 4) por domínio entre os sexos masculino e feminino, bem como o desvio padrão (dp) entre eles. Como os domínios que apresentaram melhor pontuação estão: família e amigos (média $=3,4$ ) cigarros e drogas (média=3,4), álcool (média=3,3), sono, cinto de segurança, estresse e sexo seguro (média=3,0). A melhor pontuação para o sexo masculino em relação ao feminino compreendeu somente o domínio atividade $(2,7$ e 2,4$)$ e, para o sexo feminino, envolveu cigarro e drogas $(3,5$ e 3,2$)$, álcool $(3,5$ e 3,1$)$ e trabalho/estudo $(3,0$ e 2,6$)$. 
Tabela III - Média e desvio padrão dos domínios de estilo de vida dos acadêmicos de Educação Física. Blumenau, Santa Catarina, Brasil, 2018.

\begin{tabular}{lcccc}
\hline \multirow{2}{*}{ Domínios } & \multicolumn{2}{c}{ Sexo feminino } & \multicolumn{2}{c}{ Sexo masculino } \\
& Média & dp & Média & dp \\
\hline Família e amigos & 3,4 & 0,866 & 3,3 & 0,930 \\
Atividade & 2,4 & 1,368 & 2,7 & 1,273 \\
Nutrição & 2,5 & 1,303 & 2,6 & 1,246 \\
Cigarros e drogas & 3,5 & 0,979 & 3,2 & 1,196 \\
Álcool & 3,5 & 1,047 & 3,1 & 1,356 \\
Sono, cinto de segurança, estresse e sexo seguro & 3,0 & 1,077 & 2,9 & 1,150 \\
Tipo de comportamento & 2,2 & 1,194 & 2,1 & 1,162 \\
Introspecção & 2,7 & 0,999 & 2,7 & 0,967 \\
Trabalho/estudo & 3,0 & 0,988 & 2,6 & 1,210 \\
\hline
\end{tabular}

dp: desvio padrão

A análise de variância (ANOVA) demonstrou que houve diferença entre as médias semestrais de desempenho acadêmico de acordo com a classificação de $\mathrm{EV}$, demonstrando que, quanto melhor o EV, melhor é o desempenho acadêmico.

A pontuação mais elevada do questionário EV compreendeu 95 pontos e a menor, 38 pontos. A maior média semestral envolveu 9,42 e a menor, 6,17. A Figura 1 aponta que a média semestral de desempenho acadêmico é diferente em relação à classificação do $\mathrm{EV}(\mathrm{p}=0,012)$ e que, à medida que aumentou o nível do $E V$, elevou-se a média semestral.

A Figura 2 apresenta a correlação positiva significativa entre o estilo de vida e o desempenho acadêmico estimado pela média semestral $(r=0,232 ; p=0,003)$.

Embora a correlação tenha sido considerada fraca, considerou-se significativa e diferente de zero. De forma contínua, é possível afirmar que, à medida que o EV melhora, aumenta a média semestral dos acadêmicos de Educação Física.

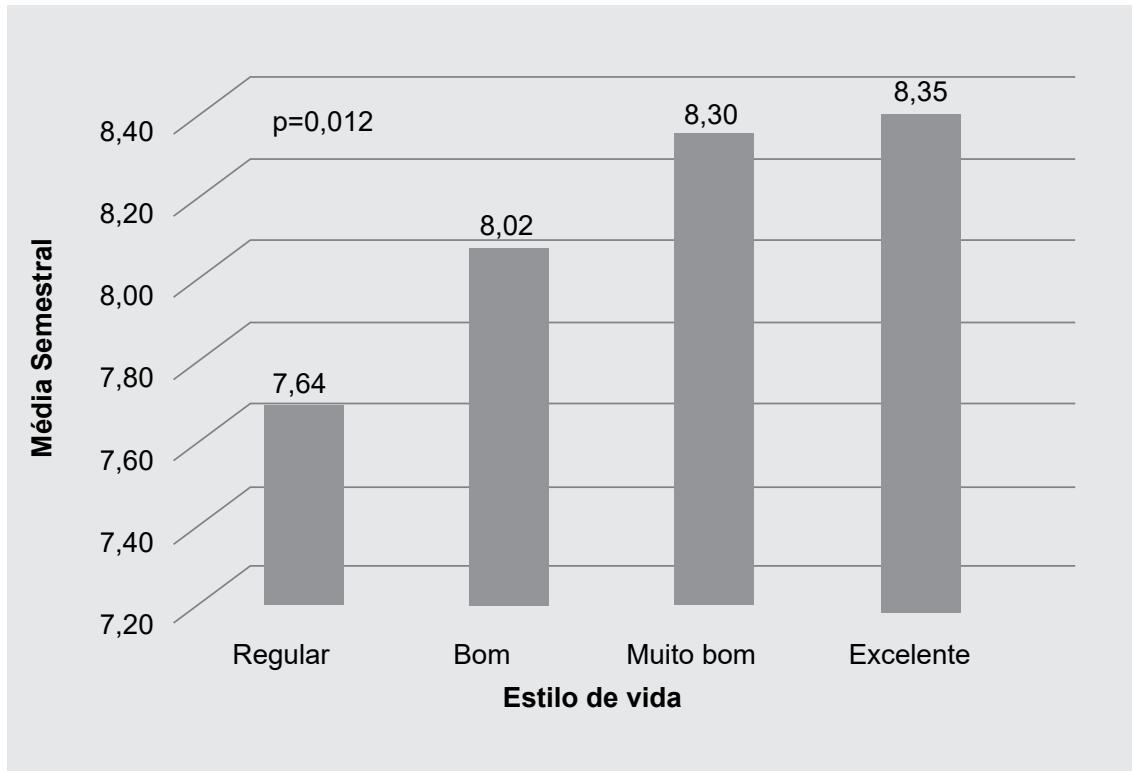

Figura 1 - Estilo de vida e desempenho acadêmico (média semestral) dos estudantes de Educação Física. Blumenau, Santa Catarina, Brasil, 2018. 


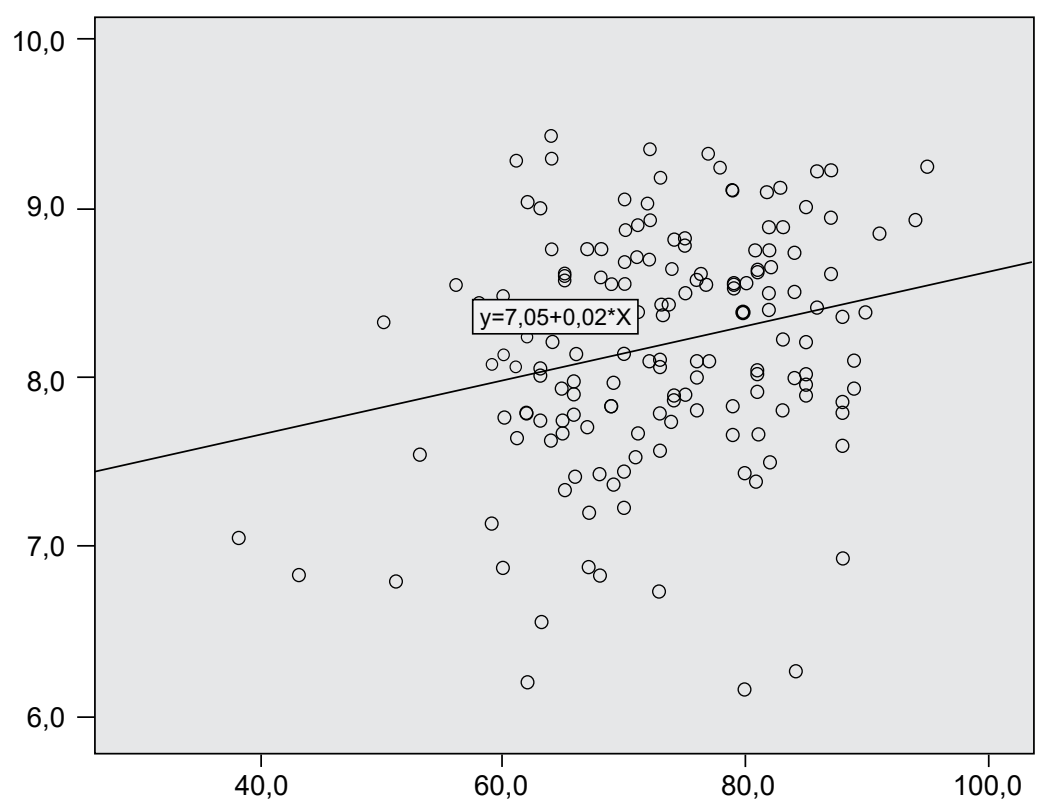

Figura 2 - Correlação entre estilo de vida e desempenho acadêmico (média semestral) dos estudantes de Educação Física. Blumenau, Santa Catarina, Brasil, 2018.

\section{DISCUSSÃO}

O ambiente universitário é um local apropriado para promover saúde. Refere-se ao período de transição da adolescência para a vida adulta, de preenchimento e busca do senso de individualidade e de construção de relações sociais. A exposição a fatores de risco comportamentais, como tabagismo, consumo excessivo de bebida alcoólica, alimentação inadequada e sedentarismo, frequentemente se inicia na adolescência e se consolida na vida adulta. Nesse sentido, ações de saúde coletiva devem promover o comportamento saudável entre os mais jovens ${ }^{(10-12)}$.

A melhora do estilo de vida de universitários por meio de ações de promoção da saúde e prevenção de doenças influencia as condições de vida, os fatores sociais e a saúde dos jovens, atualmente e no futuro. Além dos benefícios na saúde e na qualidade de vida, o estilo de vida saudável pode influenciar a produtividade e a vida universitária, o que pode se consolidar para um estilo de vida feliz ${ }^{(11,12)}$.

A mensuração do EV é complexa devido aos múltiplos fatores que o constituem e os resultados podem ser influenciados por caráter subjetivos nas respostas ${ }^{(9)}$. Estudos que associem o EV com o desempenho acadêmico em universitários ainda são escassos ${ }^{(6)}$.

Um estudo realizado com acadêmicos do curso de Enfermagem de uma instituição federal do interior do Amazonas encontrou um nível de atividade física considerada saudável e um EV global satisfatório. O nível de atividade física impactou no desenvolvimento de um EV saudável e de um bom desempenho acadêmico entre os universitários ${ }^{(6)}$.

Outro estudo, com universitários do curso de Educação Física de uma universidade pública de Sergipe, mostrou um nível de atividade física e EV inadequados. A maior parte dos alunos, 57,4\% (IC95\%: 50,7-64,0), não atendeu às recomendações para atividade física. Estudantes da licenciatura estavam mais inadequados que os do bacharelado no conjunto do EV global e nas condutas de sono, cinto de segurança, estresse e sexo seguro(7). Outra pesquisa, com acadêmicos de Educação Física de um curso à distância, apresentou como resultado geral um EV adequado, entretanto os domínios mais inadequados envolveram tipo de comportamento, nutrição e atividade física(2).

Em estudo realizado em uma instituição privada do estado do Paraná com acadêmicos de diversos cursos de graduação, os escores de EV variaram entre 63 e 74 pontos, o que classificou os estudantes avaliados entre as categorias bom e muito bom. Os autores ressaltaram a importância da orientação de estudantes universitários para um EV saudável e da disponibilização de programas de atividades físicas no ambiente acadêmico visando ao controle de peso até os últimos anos de estudo, visto serem os concluintes o grupo de maior tendência ao sobrepeso ${ }^{(11)}$.

Para o presente estudo, os escores de EV também classificaram os estudantes entre as categorias bom e muito bom de forma mais frequente, enquanto outros domínios mostraram hábitos mais frequentemente saudáveis. Por exemplo, a relação com álcool (89\% dos acadêmicos responderam nunca dirigir após beber) e o fumo $(86,6 \%$ 
dos acadêmicos responderam nunca terem fumado nos últimos cinco anos). Mesmo com uma classificação de EV satisfatório, encontraram-se domínios do questionário apresentando pontuações insatisfatórias. Para o sono, 32,4\% dos acadêmicos responderam que apenas algumas vezes dormem bem ou se sentem descansados.

Nas questões relacionadas à relação família e amigos, a maioria dos participantes, no presente estudo, obtiveram práticas saudáveis. Mais da metade dos acadêmicos relatou com frequência ou quase sempre ter com quem falar das coisas que são importantes, na mesma proporção que recebem e oferecem afeto. O convívio com novas pessoas no âmbito universitário pode ter contribuído para o elevado índice de acadêmicos que possuem alguém para conversar, dar e receber afeto. O acadêmico que possui pessoas da família, ou outras pessoas também consideradas importantes, com as quais ele possa dividir seus sentimentos, tem maiores chances de apresentar um EV adequado(2).

No presente estudo, o resultado encontrado no domínio de atividade mostrou que mais da metade dos participantes praticam quatro, cinco ou mais vezes na semana alguma atividade vigorosa por trinta minutos. Em outra pesquisa, com estudantes de Enfermagem, encontrou-se associação entre EV, atividade física e desempenho acadêmico. A prática regular de atividade física e um bom EV refletiu de maneira positiva nas notas dos acadêmicos ${ }^{(6)}$.

O presente estudo encontrou que mais da metade dos estudantes de Educação Física raramente ou até três vezes na semana praticam alguma atividade física moderada. Esse baixo nível de atividade física pode envolver algumas barreiras pessoais e sociais encontradas pelos acadêmicos, e a mais comum citada é a falta de tempo ${ }^{(7)}$. A prática de atividade física pode ser eficaz na prevenção e tratamento de várias doenças, como hipertensão e obesidade. Quando o indivíduo é pouco ativo, esses benefícios para a saúde são reduzidos ${ }^{(12)}$.

A atividade física influencia na saúde cardiovascular, na condição física e em aspectos como socialização, autoconceito e bem-estar. Além disso, cada vez mais pesquisas apontam o exercício como um fator determinante nos

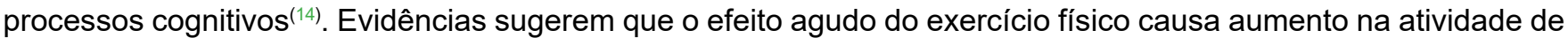
neurotransmissores. Cronicamente, o exercício pode promover adaptações em estruturas cerebrais e na plasticidade sináptica que culminariam com melhoras cognitivas ${ }^{(15)}$. Sentir prazer pela atividade física e esportiva e saber de seus benefícios à saúde pode ser o que motiva os jovens a ingressarem em um curso de Educação Física(2).

No domínio nutrição, a maioria dos acadêmicos da presente pesquisa relatou fazer uma dieta balanceada algumas vezes na semana. Relatando também, na mesma proporção, que comem em excesso açúcar e alimentos ultraprocessados. Mesmo com esse hábito não saudável, mais da metade dos acadêmicos considera que estão próximos do seu peso ideal. Resultados semelhantes encontrou-se em outros estudos, em que quase metade dos estudantes estavam com uma alimentação inadequada ${ }^{(7,11)}$.

Em geral, os acadêmicos apresentam hábitos alimentares práticos e rápidos por conta da rotina universitária, tendendo a se preocupar mais com seu desempenho acadêmico ou com suas relações sociais e lazer nas redes sociais, e esquecem-se da importância de realizar uma boa alimentação. A alimentação irregular torna possível o desenvolvimento da obesidade, diabetes, hipertensão arterial e outras doenças crônicas ${ }^{(12,16)}$.

Há evidências de que o consumo de café da manhã balanceado em crianças e adolescentes pode influenciar o desempenho cognitivo durante o aliviar da fome ou por meio dos efeitos em longo prazo pela ingestão de nutrientes que podem afetar positivamente a cognição ${ }^{(17)}$. O desempenho cognitivo e acadêmico em crianças e adolescentes está vinculado a vários indicadores de status socioeconômico, como renda familiar e educação parental. Nesse sentido, é importante considerar essas variáveis em estudos de desempenho cognitivo ou acadêmico(17).

Nos domínios álcool, cigarros e drogas, a maioria dos acadêmicos do presente estudo obteve pontuação elevada, relatando que nunca usaram cigarros ou drogas e que não abusam no consumo de bebidas alcoólicas, e aproximadamente $2 \%$ dos participantes responderam que fumam pelo menos um cigarro por dia. Os acadêmicos que responderam usar maconha ou cocaína algumas vezes são quase um quinto da amostra, considerado elevado em termos de EV saudável. Na questão sobre o álcool, 36,9\% dos acadêmicos do estudo disseram que bebem mais que quatro doses em uma única ocasião e, quase na mesma proporção, responderam que dirigem após beber.

Estudos encontraram 1,8\% dos estudantes consumindo tabaco e/ou outras substâncias, e $90,4 \%$ dos estudantes de Educação Física relataram ter feito uso de álcool alguma vez na vida, enquanto 1,1\% dos estudantes de Educação Física consumiam álcool diariamente. Encontrou-se, também, um elevado consumo de anabolizantes $(7,9 \%)$ e de maconha $(25,2 \%)$ pelos alunos de Educação Física ${ }^{(7,18,19)}$.

Um estudo relata que grande parte dos fumantes inicia esse hábito ao entrar na universidade ${ }^{(7)}$. No Brasil, o uso regular de bebidas alcoólicas começa aos 17 anos, ou seja, muito próximo ao ingresso na universidade (2). Os estudantes da área da saúde são um grupo que merece atenção, uma vez que, no futuro, poderão trabalhar com essa temática e são os que mais se envolvem em abuso de álcool(20), a droga mais consumida entre os jovens e 
um dos mais importantes fatores de risco para a adoção de outras condutas de risco para a saúde ${ }^{(2,20)}$. Diversos fatores podem influenciar o consumo de bebidas alcoólicas, como o contexto familiar e social, o poder aquisitivo e a facilidade de acesso $^{(2)}$.

Problemas físicos, psicológicos e sociais são as consequências do consumo de drogas e álcool entre universitários. Entre eles, estão: acidentes automobilísticos, violência, comportamento sexual de risco, prejuízos acadêmicos, diminuição de percepção e estresse. O consumo dessas substâncias leva a problemas psicológicos e comportamentais, incluindo comprometimento cognitivo. O mau desempenho acadêmico pode ser a principal consequência desse comprometimento cognitivo, que pode ocorrer devido às faltas, aos atrasos e à perda de atenção ou sonolência em sala de aula(21).

O domínio sono, cinto de segurança e sexo seguro apresentou maior pontuação média no presente estudo, o que contribuiu como fator positivo para EV saudável. Um tema que merece atenção é o sono. Mais da metade dos acadêmicos respondeu que raramente ou algumas vezes dorme bem ou se sente descansado. Para outro estudo, $51 \%$ dos estudantes da área da saúde, além de uma qualidade de sono ruim, apresentam sonolência diurna. Esses estudantes podem ter uma maior carga horária em sala de aula e envolvimento em atividades extracurriculares, podendo ocasionar alterações do padrão do sono ${ }^{(22)}$. Acredita-se que a privação de sono pode estar diretamente associada ao seu decréscimo e influenciar o processo de aprendizagem ${ }^{(23)}$.

Em outro estudo encontrou-se que os universitários tendem a apresentar um padrão de sono irregular por atrasos de início e final do sono, por curta duração de sono nos dias de semana e longa duração de sono nos finais de semana. Como consequência dessa irregularidade, acadêmicos apresentavam qualidade de sono ruim e baixo desempenho acadêmico ${ }^{(24)}$. O uso de cinto de segurança $(10 \%)$ e o sexo seguro $(55,7 \%)$ são negligenciados ${ }^{(7)}$.

Encontrou-se, no presente estudo, um resultado controverso relativo ao alto índice dos acadêmicos que sempre usam cinto de segurança e mais da metade sempre praticam relação sexual com uso de preservativo. Estudantes com elevado desempenho acadêmico se preocupam com a prevenção, afirmando realizar sexo seguro e fazer o uso de cinto de segurança. Além disso, estão menos propensos a desenvolverem o hábito de fumar, demonstrando que comportamentos preventivos também podem estar relacionados a um elevado desempenho acadêmico(6).

No presente estudo, para o tipo de comportamento e introspecção, mais de um terço dos acadêmicos apresenta estar com pressa, com sentimentos de raiva ou se sentindo hostis algumas vezes ou com relativa frequência, quase na mesma proporção com que se sentem tensos ou tristes.

Em estudo de acompanhamento do EV dos acadêmicos de uma universidade do Recôncavo Baiano, os resultados demonstraram que faz parte do cotidiano dos universitários a pressa e os sentimentos de raiva. Esses sentimentos podem existir devido à ansiedade e angústia pelas cobranças constantes de um bom desempenho acadêmico. Em relação à introspecção, os acadêmicos que ingressaram na universidade com esses níveis baixos apresentaram maiores chances de mantê-los após dois anos na graduação(25).

Pensar de forma positiva e otimista, assim como não se sentir raivoso, triste ou deprimido, apresenta forte relação com o EV adequado. Na fase universitária, existe um aumento no interesse por novos conhecimentos e experiências, e é normal a exploração das capacidades pessoais em busca da autonomia, do amor e da amizade ${ }^{(2)}$.

O presente estudo encontrou que mais da metade dos acadêmicos considera que está com relativa frequência ou quase sempre satisfeito com o trabalho/estudo que exerce. Nesse caso, há satisfação com o curso de Educação Física, com os estágios e com trabalho na área. Resultado diferente de outro estudo( ${ }^{(7)}$, no qual quase um sexto dos participantes declarou insatisfação com o trabalho, o que poderia impactar no desempenho acadêmico.

Obtiveram-se os dados por meio de questionário autoaplicável, passível de viés de memória, o que pode ser considerado uma limitação do atual estudo.

\section{CONCLUSÃO}

O estilo de vida dos estudantes investigados se associou ao desempenho acadêmico, sendo seu estilo de vida classificado com maior frequência em bom e muito bom. Observando de forma isolada os domínios, percebe-se que família e amigos; cigarros e drogas; álcool, sono, cinto de segurança, estresse e sexo seguro; e trabalho/estudo apresentaram pontuação satisfatória para um estilo de vida saudável.

\section{CONFLITOS DE INTERESSE}

Os autores informam que não há conflitos de interesses. 


\section{CONTRIBUIÇÕES}

Cristiani Devalieri e Clóvis Arlindo de Sousa contribuíram com a elaboração e delineamento do estudo; a análise e interpretação dos dados; e a redação e/ou revisão do manuscrito. Quelen Schutz Carvalho Bernardes Malafaia e Maiara Vargas Schmitt contribuíram com a redação e/ou revisão do manuscrito.

\section{REFERÊNCIAS}

1. World Health Organization. Healthy living: what is a healthy lifestyle? [Internet]. Copenhagen: WHO Regional Office for Europe; 1999 [acesso em 2019 Nov 04]. Disponível em: https://apps.who.int/iris/ handle/10665/108180

2. Vargas LM, Redkva PE, Cantorani JRH, Gutierrez GL. Estilo de vida e fatores associados em estudantes universitários de Educação Física. Rev Atenção Saúde. 2015;13(44):17-26.

3. Sousa CA, Nunes CRO. Estilo de Vida Saudável e Saúde Coletiva. Blumenau: Edifurb; 2015.

4. Fonsêca PN. Desempenho acadêmico de adolescentes: proposta de um modelo explicativo [tese]. Paraíba: Universidade Federal da Paraíba; 2008.

5. Miranda GJ, Lemos KCS, Oliveira AS, Ferreira MA. Determinantes do desempenho acadêmico na área de negócios. Rev Meta Aval. 2015;7(20):175-209.

6. Souza KJQ, Borges GF. Estilo de vida, atividade física e coeficiente acadêmico de universitários do interior do Amazonas-Brasil. Rev Bras Ciênc Saúde. 2016;20(4):277-84.

7. Silva DAS, Pereira IMM, Almeida MB, Silva RJS, Oliveira ACC. Estilo de vida de acadêmicos de Educação Física de uma universidade pública do estado de Sergipe, Brasil. Rev Bras Ciênc Esporte. 2012;34(1):53-67.

8. Belem IC, Camargo DAD, Both J. Diferentes comportamentos do estilo de vida de Universitários do curso de Educação Física. Rev Cienc Ejerc Salud. 2019;17(2):1-15.

9. Añez CRR, Reis RS, Petroski EL. Versão brasileira do questionário "Estilo de Vida Fantástico": tradução e validação para adultos jovens. Arq Bras Cardiol. 2008;91(2):101-9.

10. Berbigier MC, Magalhães $C R$. Educação nutricional em universitários e estratégias para promoção de saúde institucional: revisão integrativa. Rev Bras Promoç Saúde. 2017;30(2):264-74.

11. Santos JJA, Saracini N, Silva WC, Guilherme JH, Costa TA, Silva MRAG. Estilo de vida relacionado à saúde de estudantes universitários: comparação entre ingressantes e concluintes. Abcs Health Sci. 2014;39(1):1723.

12. Luna AA, Molari M, Primo SH, Bispo NNC, Ossada VAY, Costa VSP. Caracterização do estilo de vida de universitários do ensino superior a distância. J Health Sci. 2018;20(1):40-4.

13. Instituto Brasileiro de Geografia e Estatística. Blumenau: população [Internet]. 2019 [acesso em 2019 Nov 02]. Disponível em: https://cidades.ibge.gov.br/brasil/sc/blumenau/panorama

14. Reloba S, Chisosa LJ, Reigal RE. Relacíon entre actividad física, procesos cognitivos y rendimiento académico de escolares: revisión de la literatura actual. Rev Andal Med Deporte. 2016;9(4):166-72.

15. Merege CAA Filho, Alves CRR, Sepúlveda CA, Costa AS, Lancha AH Jr, Gualano B. Influência do exercício físico na cognição: uma atualização sobre mecanismos fisiológicos. Rev Bras Med Esporte. 2014;20(3):23741.

16. Tassini CC, Val GR, Candido SS, Bachur CK. Assessment of the Lifestyle of University Students in the Healthcare Area Using the Fantastic Questionnaire. Int J Cardiovasc Sci. 2017;30(2):117-22.

17. Rampersaud GC, Pereira MA, Girard BL, Adms J, Metzl JD. Breakfast habits, nutritional status, body weight, and academic performance in children and adolescents. J Am Diet Assoc. 2005;105(5):743-60.

18. Fernandes TF, Monteiro BMM, Silva JBM, Oliveira KM, Viana NAO, Gama CAP, et al. Uso de substâncias psicoativas entre universitários brasileiros: perfil epidemiológico, contextos de uso e limitações metodológicas dos estudos. Cad Saúde Coletiva. 2017;25(4):498-507. 
19. Chiapetti N, Serbena CA. Uso de álcool, tabaco e drogas por estudantes de uma universidade particular da cidade de Curitiba: fatores relacionados à história de uso e prevalência de consumo. Psicol Reflex Crit. 2007;20(2):303-13.

20. Cardoso FM, Barbosa HA, Costa FM, Vieira MA, Caldeira AP. Fatores associados à prática do binge drinking entre estudantes da área da saúde. Rev Cefac. 2015;17(2):475-84.

21. Trindade BPA, Diniz AVJAR. Uso de drogas entre estudantes universitários: uma perspectiva nacional. Rev Med Saúde Brasília. 2018;7(1):52-60.

22. Carvalho TMCS, Silva II Jr, Siqueira PPS, Almeida JO, Soares AF, Lima AMJ. Qualidade do sono e sonolência diurna entre estudantes universitários de diferentes áreas. Rev Neurocienc. 2013;3(21):383-7.

23. Furlani R, Ceolim MF. Padrões de sono de estudantes ingressantes na Graduação em Enfermagem. Rev Bras Enferm. 2005;58(3):320-4.

24. Almondes KM, Araujo JF. Padrão do ciclo sono-vigília e sua relação com a ansiedade em estudantes universitários. Estud Psicol (Natal). 2003;8(1):37-43.

25. Brito BJQ, Gordia AP, Quadros TMB. Estilo de vida de estudantes universitários: estudo de acompanhamento durante os dois primeiros anos do curso de graduação. Medicina (Ribeirão Preto). 2016;4(49):293-302.

\section{Endereço para correspondência:}

Quelen Schutz Carvalho Bernardes Malafaia

Universidade Regional de Blumenau - FURB

Rua São Paulo, 2171/ Campus 3 - Sala 302

Bairro: Itoupava Seca

CEP: 89.030-001 - Blumenau - SC - Brasil

E-mail: quelenschutz@gmail.com

Como citar: Malafaia QSCB, Devalieri C, Schmitt MV, Sousa CA. Relação entre estilo de vida e desempenho acadêmico. Rev Bras Promoç Saúde. 2019;32:9719. 